\title{
Erratum to: Nasal pressure recordings for automatic snoring detection
}

\author{
Hyo-Ki Lee ${ }^{1}$ Hojoong Kim² $•$ Kyoung-Joung Lee ${ }^{3}$
}

Published online: 27 October 2016

(C) International Federation for Medical and Biological Engineering 2016

Erratum to: Med Biol Eng Comput (2015)

53:1103-1111

DOI 10.1007/s11517-015-1388-2

In the original publication of the article, Fig. 4 was incorrect. The correct Fig. 4 is given here.

The online version of the original article can be found under doi:10.1007/s11517-015-1388-2.

Kyoung-Joung Lee

1kj5809@yonsei.ac.kr

1 Interdisciplinary Consortium on Advanced Motion

Performance (iCAMP), Department of Surgery,

College of Medicine, The University of Arizona,

Tucson, AZ 85724, USA

2 Division of Pulmonary and Critical Care Medicine,

Samsung Medical Center, Sungkyunkwan University

School of Medicine, 81 Irwon-ro, Gangnam-gu,

Seoul 06351, Republic of Korea

3 Department of Biomedical Engineering, Yonsei University,

1 Yonseidae-gil, Wonju-si, Gangwon-do 26493,

Republic of Korea 
Fig. 4 Example of real-time automatic snoring detection using nasal pressure recording

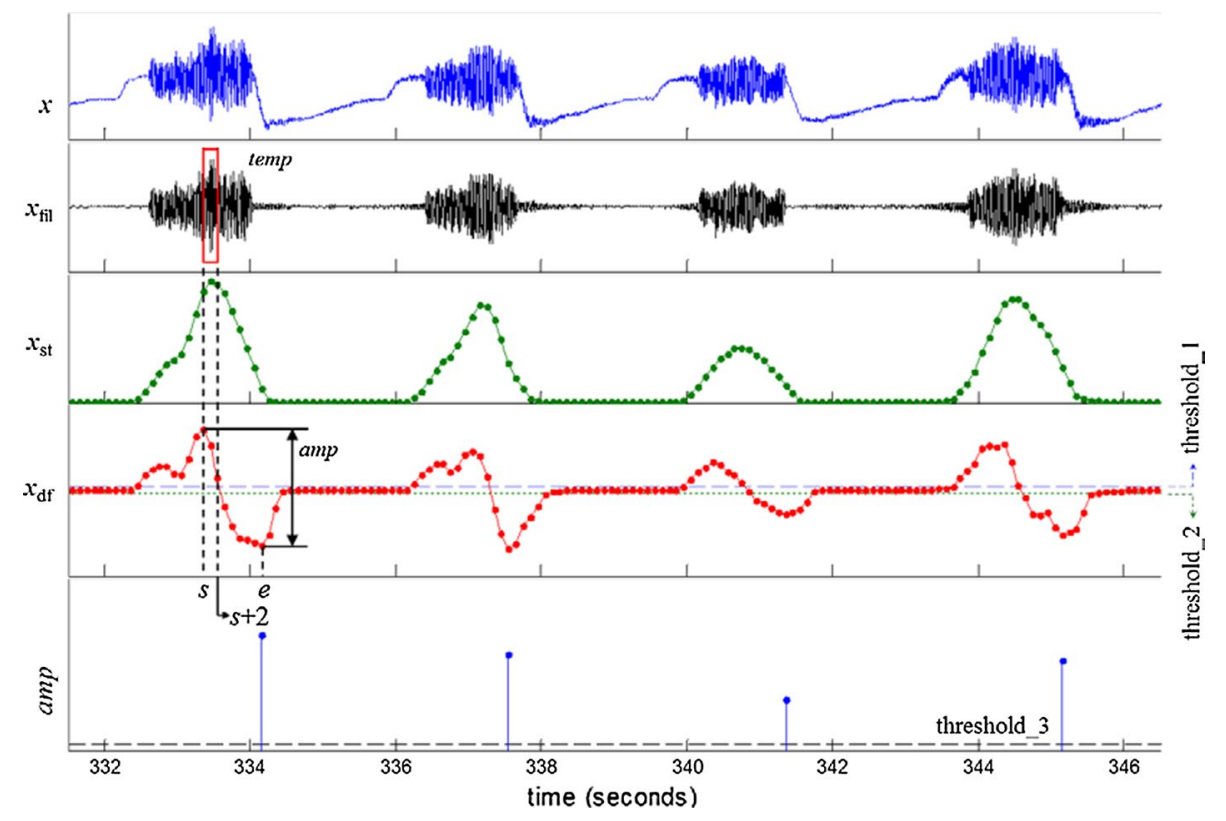

